\title{
Perinatal Oral Health: A Novel Collaborative Initiative to Improve Access, Attitudes, Comfort Level, and Knowledge of Pregnant Women and Dental Providers
}

\author{
${ }^{1}$ Division of Practice Essentials and Interprofessional Education, \\ University of Detroit Mercy School of Dentistry, Detroit, Michigan \\ ${ }^{2}$ Division of Maternal Fetal Medicine, Department of Obstetrics and \\ Gynecology, Wayne State University School of Medicine, Detroit, \\ Michigan \\ ${ }^{3}$ A. T. Still University Arizona School of Dentistry \& Oral Health, Mesa,
}

Melanie E. Mayberry, DDS, MS-HCM ${ }^{1} \quad$ Bernard Gonik, MD² Robert M. Trombly, DDS, JD ${ }^{3}$ Arizona

Am J Perinatol Rep 2020;10:e54-e61.
Address for correspondence Melanie E. Mayberry, DDS, MS-HCM, University of Detroit Mercy School of Dentistry, 2700 Martin Luther King Jr. Blvd., Detroit, MI 48208 (e-mail: mayberme@udmercy.edu).

\begin{abstract}
Objectives The objectives of this program were to increase access to dental care among pregnant women and to improve dental students' exposure, comfort level, and knowledge of the potential impact of poor oral health on pregnancy outcomes.

Study Design Through collaborative efforts of a School of Dentistry and a School of Medicine, the Oral Health Pregnancy Day Initiative (OHPDI) was developed. Dental students were educated on the impact poor oral health may have on pregnancy outcomes and the importance of access to care. Pregnant women received perinatal oral health education and needed dental care.

Results Thirty-four pregnant women presented for the OHPDI. Thirty-nine dental students participated. Eighty-five percent of students reported they learned how poor oral health may have a negative impact on pregnancy and birth outcomes; $79 \%$ agreed as a result of the event they were more likely to treat pregnant women. Ninety-four percent of pregnant women reported not having a dentist and $100 \%$ received perinatal

Keywords

- perinatal care

- dental students

- dentists

- interprofessional oral health education and needed dental care. Eighty-eight pregnant women were seen subsequent to the OHPDI.

Conclusion This initiative resulted in increased students' knowledge, exposure, and comfort level to treating pregnant women and pregnant women received needed oral health care education and dental treatment.
\end{abstract}

This article presents a novel pilot project to initiate change in knowledge, perception, and behavior of pregnant women and dental students. There have been no documented studies of initiatives such as this project that demonstrates collaboration of a dental education clinical program with obstetrics, free dental treatment to pregnant women, enhanced educational opportunities for dental students and patients, and dedicated care to pregnant women.

received

August 9, 2019

accepted after revision

November 15, 2019
DOI https://doi.org/

10.1055/s-0040-1702927. ISSN 2157-6998.
The initiative described in this chapter sought to increase exposure of dental students to the treatment of pregnant women, facilitate promotion and awareness of the 2013 American College of Obstetricians Gynecologists guideline, ${ }^{1}$ and the 2012 National Consensus Statement, ${ }^{2}$ increase exposure and comfort to treating pregnant patients, and create opportunities for pregnant women to receive needed dental care and oral health education.

Copyright $\odot 2020$ by Thieme Medical Publishers, Inc., 333 Seventh Avenue, New York, NY 10001, USA. Tel: +1(212) 760-0888
License terms

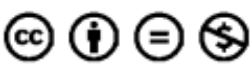


Misinformation regarding the safety and importance of oral health care treatment for pregnant women and their unborn child exist among patients and providers. ${ }^{3}$ Health literacy plays a role among patients. ${ }^{3}$ Many are unaware of the potential negative impact of poor oral health on preterm birth, low birth weight, pregnancy outcomes, and early childhood caries. ${ }^{3,4}$ While social determinants of health also impacts dental care utilization for pregnant women, ${ }^{5}$ a study in Michigan showed that poor patient knowledge and understanding of perinatal oral health crosses demographic boundaries. $^{6}$

Reluctance to treat attitudes of dentists can have a significant negative impact on current practice ${ }^{3}$ as dentists play key roles in the health of pregnant women. ${ }^{4,7}$ Many US studies examining dentists' attitudes regarding treating pregnant women report several dentists' practice patterns are not consistent with current guidelines and recommendations. Thirteen states and national organizations have published perinatal oral health guidelines to reduce barriers to care and increase oral/dental care utilization during pregnancy. ${ }^{8}$ Thus, it is important to provide training and exposure in dental schools. In 2013, Curtis et al suggested that future efforts of prenatal oral health education in US dental schools should include increasing exposure of dental students to pregnant patients. ${ }^{9}$

Despite national recommendations and guidelines, utilization of care during pregnancy is reported as low. ${ }^{5}$ Obstetricians play an important role in advancing oral health and dental treatment for pregnant patients. A study involving obstetricians and gynecologists reported $77 \%$ of their pregnant patients were "declined" treatment by dentists. ${ }^{10}$ Pregnancy represents a time of increased risk to dental pathology. ${ }^{5}$ Delaying dental treatment due to pregnancy may worsen dental outcomes and the health of the mother and child. ${ }^{4}$ Poor maternal oral health may lead to infection, pain, and unnecessary loss of teeth. ${ }^{4}$ It may also lead to early childhood caries in the child ${ }^{11}$ due to the transfer of cariogenic bacteria from mother to infant child. ${ }^{12}$ Additionally, maternal oral health is a public health issue because poor maternal dental health may increase the risk of early childhood dental caries, which among young children is the most chronic condition in the world. Maternal dental disease may increase the risk of miscarriage, preterm birth, and fetal growth restriction. ${ }^{1}$ Evidence is mounting that oral bacteria play a role in intrauterine infection. ${ }^{13}$ The association of periodontal disease with low birth weight and preterm birth has been well established. ${ }^{14,15}$ In the 2018, Rangel-Rincon et al systematic/meta-analysis review of periodontal intervention on reducing adverse pregnancy outcomes, six studies showed low positive effect on periodontal intervention on adverse pregnancy outcomes while 11 demonstrated moderate-to-high positive effect on periodontal intervention reducing adverse pregnancy outcomes. ${ }^{16}$ It has also been noted that periodontal disease intervention during pregnancy has not consistently resulted in reduction in preterm birth and low birth weight events. ${ }^{14,15}$ Emerging evidence suggests periodontal intervention prior to pregnancy may reduce adverse pregnancy outcomes. ${ }^{15}$

\section{Methods}

\section{Overview}

This study was approved by the Institutional Review Board at the University of Detroit Mercy on July 14, 2015, IRB number 1415-83.

A dental faculty member from the University of Detroit Mercy School of Dentistry and a medical faculty member from the Obstetrics \& Gynecology/Maternal and Fetal Medicine Department of Wayne State University Medical School in Detroit engaged in an interprofessional collaboration to create the School of Dentistry's first Oral Health Pregnancy Day Initiative (OHPDI) held over two separate days due to patient demand. The OHPDI occurred during normal clinic hours and oral health care treatments were supervised by faculty normally assigned to the clinic. Both events occurred on Fridays, one day in March and another day in April. Both schools have clinics that are co-located on the Detroit Medical Center Campus.

\section{Pregnant Women Participation Criteria}

All pregnant women qualified for the event with the presentation of a medical consult from their $\mathrm{OB}$ provider indicating current pregnancy status. Patients learned of the event through radio advertisement, community care coordinators, social workers, and local health care providers. Patients requiring treatment beyond the day of the events were encouraged to return for continuation of care, free of charge. Patients who presented for the OHPDI were registered in similar fashion as any patient seeking dental care at the School of Dentistry. The length of time each patient spent varied based on the time the patient presented to the clinic for treatment, dental treatment needs identified, and patients' personal time schedule. Thirty-four women sought care at this event. The maximum number that could be seen per day was $\sim 78$ patients. All women that presented were seen. Care was provided on a first come first served basis.

\section{Dental Students Participation Criteria}

The dental class size for each class varies between 141 and 144 . At the time of this event, a maximum of 40 students could have participated due to the number of dental clinic chairs available in this clinic. Assigned students were expected to see pregnant patients. Student clinic assignments were scheduled randomly several months in advanced. Thirty-nine third- and fourthyear dental students participated. Some participated due to their normal clinical assignment at the 40-chair hospitalbased dental clinic. Others, third-year students in particular, volunteered to participate through their oral medicine course. All the dental chairs were reserved for this event. Each of the participating students provided care for one woman and a few students may have shared a patient. Student availability varied both days of the event due to student scheduling. Assigned students were available all day to see patients.

\section{Dental Students and Pregnant Women Perinatal Oral Health Education}

Dental students were educated on the potential negative impact poor oral health may have on pregnancy and birth 
outcomes. Faculty from dental and obstetrics were present. Supervised by faculty, dental students treated pregnant women. Pregnant patients were educated on the importance of oral health for them and their baby and the negative impact poor oral health may have on pregnancy and birth outcomes.

\section{Oral Health/Dental Treatment Provided}

Oral health care treatment was provided free of charge through the generous grant of the Detroit Medical Center Foundation (grant \# 2015-0098). Due to the number of students available to treat patients and the number of patients who presented for treatment, there was minimal wait time for patients to be seen. Treatment provided to pregnant patients was based on oral health exams and radiographic assessments. Treatment provided during the OHPDI included perinatal oral health education, oral health examinations, radiographic assessments, prophylaxis, gross debridements, fluoride treatments, amalgam, and composite fillings. All procedures including health education and preventive services were performed by dental students.

Thirty-four pregnant patients received oral health examinations, radiographic assessments as needed, and oral health education and treatment over the 2-day event. Patients requiring additional services were encouraged to return for continuation of care.

\section{Dental Students Survey Assessment Post Participation} Post event paper surveys were presented to participating students. The survey was developed and designed by author MM and reviewed by faculty for clarity and content and pilot tested with a small sample of nonparticipating students. Feedback was used to revise the survey and to develop the final version. Similar questions have been used in other reported studies. ${ }^{17}$ Surveys were administered to students immediately after the OHPDI. Surveys were confidential and kept secured in a locked file cabinet. The instrument included seven questions with question \#4 as a four-part Likert agreement scale question (-Table $\mathbf{1}$ ). The survey response rate for the participating dental students was $100 \%$. However, some students did not answer all survey questions.

\section{Pregnant Women Survey Assessment Post Participation}

Post event surveys written in English were also collected from patients. The survey was developed and designed by author MM and reviewed by faculty for clarity and content. Feedback was used to revise the survey and develop the final version. The questions were designed to specifically assess the goals of the grant initiative of increasing awareness and education of oral health among pregnant women and providing opportunities to receive needed dental care. The eight-question survey was administered to patients immediately after the OHPDI (-Table 2). Written informed consents were obtained. The response rate for the pregnant women was $100 \%$.

\section{Data Analysis}

Data was imported into Qualtrics survey. Univariate descriptive statistics were used to analyze the findings. Due to the sample size, this was regarded as a pilot project.

\section{Results}

During the 2015 to 2016 academic year, two OHPDIs occurred. The descriptive survey findings of this cross-sectional design are shown in the following figures. Thirty-nine students participated and thirty-four pregnant patients were treated during the OHPDI. The survey was confidential and did not allow stratification of students into third- or fourth-year students. However, due to normal clinic assignments for this clinic, it can be assumed the majority were fourth-year students. Eleven students (28\%) reported never having treated a pregnant patient prior to the OHPDI. Fourteen students (36\%) reported neutrality or uncomfortable treating pregnant patients (-Fig. 1). As a result of the OHPDI, 33 (85\%) agree/strongly agree they learned how poor oral health can negatively impact pregnancy and birth outcomes (-Fig. 2), 28 (72\%) agree/strongly agree they are more likely to treat pregnant patients during any trimester not just the second trimester, 34 (87\%) students agree/strongly agree they are more comfortable treating pregnant patients, and $31(79 \%)$ agree/strongly agree they are more likely to treat pregnant patients in their practice after graduation, and 34 (87\%) agree/strongly agree they are comfortable treating

Table 1 Dental students survey assessment questions post OHPDI participation

\begin{tabular}{|l|l|}
\hline Survey Questions & Response Choices \\
\hline 1. To your knowledge have you ever treated a pregnant patient? & Choices provided \\
\hline $\begin{array}{l}\text { 2. Prior to today's Oral Health and Pregnancy Day Initiative please rate your } \\
\text { comfort of treating pregnant patients. }\end{array}$ & Likert scale \\
\hline 3. How comfortable did your patient seem to be receiving dental treatment? & Likert scale \\
\hline 4a. I learned how poor oral health may negatively impact pregnancy and birth outcomes. & Likert scale \\
\hline 4b. I am more likely to treat pregnant patients during any trimester, not just the second. & Likert scale \\
\hline 4c. I am more likely to treat pregnant patients in my office after graduation. & Likert scale \\
\hline 4d. I am comfortable treating pregnant patients. & Likert scale \\
\hline
\end{tabular}

Abbreviation: OHPDI, Oral Health Pregnancy Day Initiative. 
Table 2 Pregnant women survey assessment questions post OHPDI participation

\begin{tabular}{|l|l|}
\hline Survey Questions & Response Choices \\
\hline 1. Please indicate your age range. & Choices provided \\
\hline 2. Please indicate the number of months you are pregnant. & Choices provided \\
\hline 3. How did you hear about this event? & Choices provided \\
\hline 4. Do you have a dentist? & Choices provided \\
\hline 5. Prior to today, when did you last see a dentist? & Choices provided \\
\hline 6. Have you ever gone to an emergency room for dental care? & Choices provided \\
\hline 7. Today's oral health pregnancy day initiative taught me that oral health is important for me. & Likert Scale \\
\hline 8. Today's oral health pregnancy day initiative taught me that oral health is important for my pregnancy. & Likert Scale \\
\hline 9. Because of today's oral health pregnancy day initiative I will more likely take care of my oral health. & Likert Scale \\
\hline $\begin{array}{l}\text { 10. Because of today's oral health pregnancy day initiative I will more likely take care of my baby's oral } \\
\text { health. }\end{array}$ & Likert Scale \\
\hline $\begin{array}{l}\text { 11. Because of today's oral health pregnancy day initiative I will more likely seek comprehensive } \\
\text { dental care for me. }\end{array}$ & Likert Scale \\
\hline $\begin{array}{l}\text { 12. Because of today's oral health pregnancy day initiative I will more likely seek preventative dental } \\
\text { care for me. }\end{array}$ & Likert Scale \\
\hline $\begin{array}{l}\text { 13. Because of today's oral health pregnancy day initiative I will more likely seek comprehensive } \\
\text { dental care for my baby. }\end{array}$ & Likert Scale \\
\hline $\begin{array}{l}\text { 14. Because of today's oral health pregnancy day initiative I will more likely seek comprehensive } \\
\text { dental care for my baby. }\end{array}$ & Likert Scale \\
\hline
\end{tabular}

Abbreviation: OHPDI, Oral Health Pregnancy Day Initiative.

pregnant patients. Thirty-two (82\%) indicated their patients were comfortable during dental treatment (-Fig. 3 ).

Regarding pregnant patients, two(6\%) patients ranged in age between 16 and 20 years old, $12(35 \%)$ ranged from 21 to
25 years old, $11(32 \%)$ ranged 26 to 30 years old, $6(18 \%)$ ranged from 31 to 35 years old, $3(9 \%)$ ranged over 36 years old, $1(2 \%)$ patient did not indicate the number of months she was pregnant, $9(26 \%)$ indicated they were 1 to 3 months

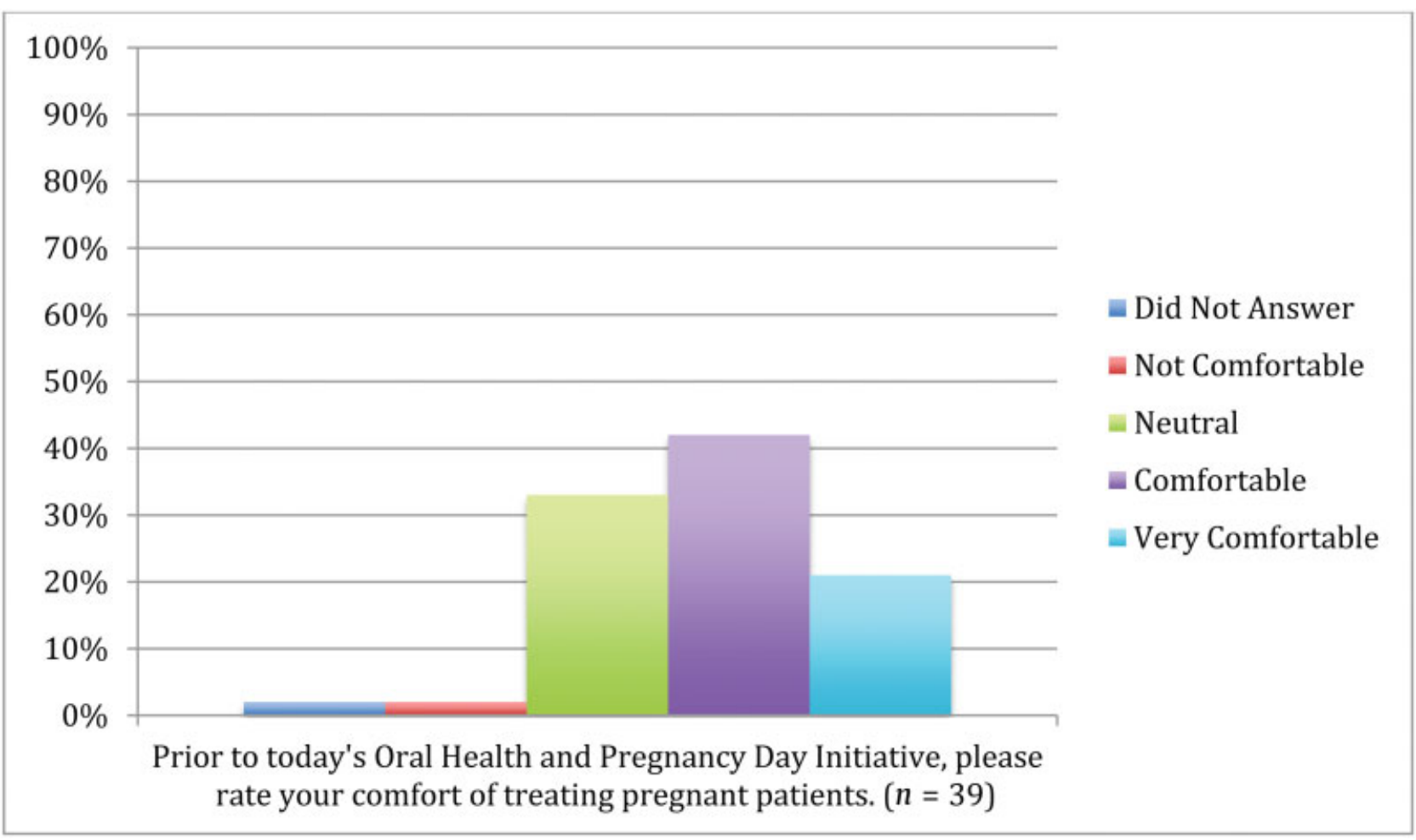

Fig. 1 Dental student comfort level of treating pregnant patients prior to the Oral Health Pregnancy Day Initiative. 
I learned how poor oral health can negatively impact pregnancy and birth outcomes. $(n=39)$

I am more likely to treat pregnant patients during any trimester, not just the second.

$$
(n=39)
$$

I am more likley to treat pregnant patients in my office after graduation. $(n=39)$

I am comfortable treating pregnant patients $(n=39)$

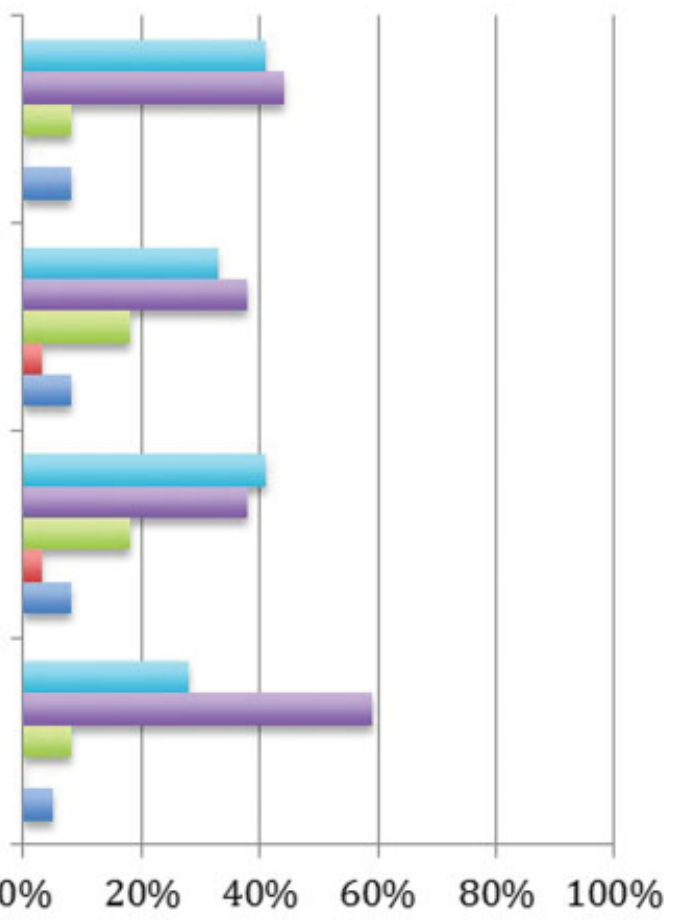

= Strongly agree = Agree

N Neutral

ఐDisagree

ఐStrongly disagree

Fig. 2 Level of student agreement to the impact of the Oral Health Pregnancy Day Initiative.

pregnant, 21(61\%) indicated they were 3 to 6 months pregnant, and $3(8 \%)$ indicated they were greater than 6 months pregnant. All 34 women received oral health education from the students, 32(94\%) reported not having a dentist, 22(65\%) reported being over a year since they last saw a dentist, 30(88\%) agreed/strongly agreed that OHPD taught that "oral health is important for me" and that "oral health is important for pregnancy," 32(94\%) agreed/strongly agreed that they will more likely take care of their oral health, 31(91\%) agreed/strongly agreed that they will more

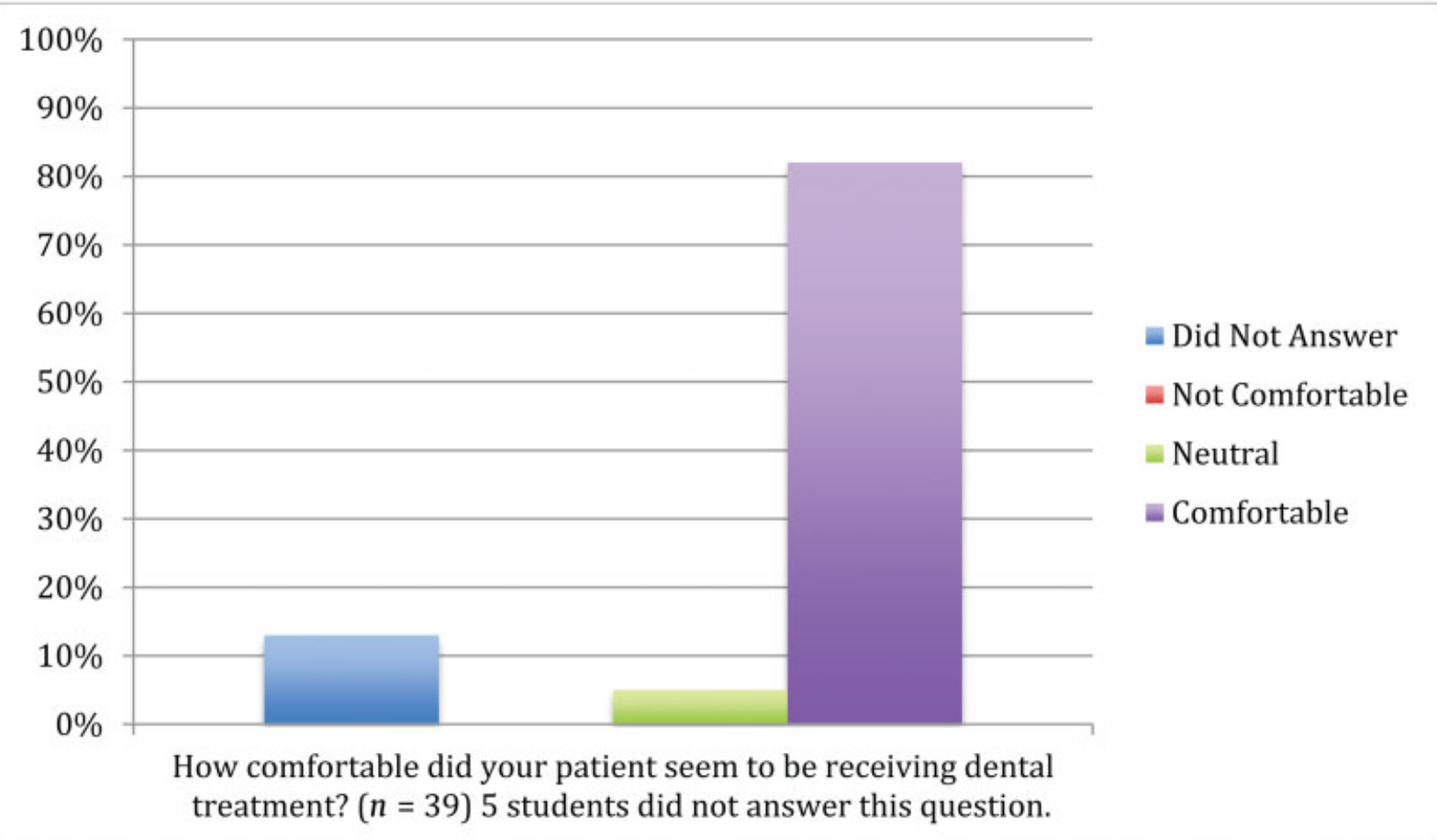

Fig. 3 Comfort level of pregnant women during dental treatment. 


\section{Regarding Oral Health Pregnancy Day Initiative $N=34$}

I will seek preventive dental care for my baby.

I will seek comprehensive dental care for my baby.

I will seek preventive dental care for myself.

I will seek comprehensive dental care for myself.

I will more likely take care of my baby's oral health

I will more likely take care of my oral health.

Taught me oral health is important for pregnancy.

Taught me that oral health is important for me.

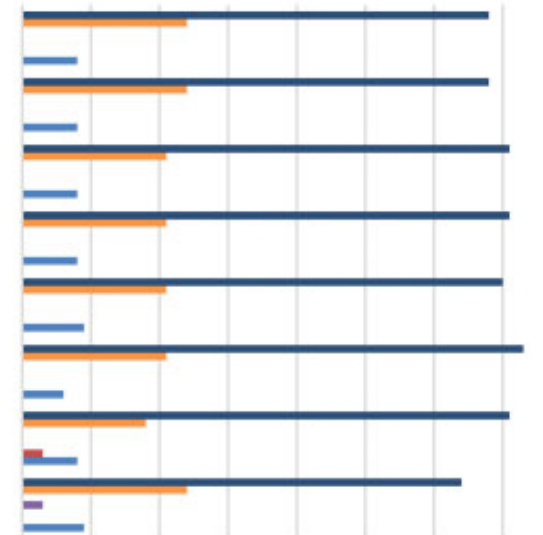

0\% $10 \%$ 20\% 30\% $40 \%$ 50\% $60 \%$ 70\% $80 \% 90 \% 100 \%$

- Strongly Agree $\quad$ Agree
= Somehwat Disagree $=$ Disagree

- Somewhat Agree $\quad$ Neutral
= Strongly Disagree

Fig. 4 Level of pregnant women agreement to the impact of the Oral Health Pregnancy Day Initiative.

likely take care of their baby's oral health, 31(91\%) agreed/strongly agreed that they will seek comprehensive dental care and preventive for themselves, 31(91\%) agreed/strongly agreed that they will seek comprehensive dental care and preventive dental care for their baby (-Fig. 4). Recognizing that some patients may demonstrate response bias, it was more logistically feasible for the student providing the care to administer and collect the surveys. Front desk staff also collected the surveys. No pretest survey was administered.

The distribution of treatment rendered during the OHPDI event was 34 perinatal oral health education and oral hygiene instructions, 31 exams, 7 radiographs, 21 adult prophylaxis or gross debridements, 22 fluoride applications, and 2 extractions. Students informed the patients of the recommended treatment needed. Patients were given the option to accept or decline treatment.

It is difficult to determine how many patients accepted and completed all the recommended treatment. However, the OHPDI event promoted awareness of the importance of perinatal oral health, safety of dental treatment, intentional efforts for dental students to treat pregnant women, and intentional efforts for pregnant women to receive needed dental care. It served as a gateway for more pregnant women to receive treatment. Because of the OHPDI, 88 pregnant women were seen after the OHPDI event and $20(59 \%)$ of the patients were initially seen during the OHPDI event and returned for continuation of treatment, 63 radiographic assessments, 23 adult prophylaxis, 9 gross periodontal debridements, 15 scaling and root planning periodontal therapies, 35 fluoride applications, 10 endodontic pulp therapies (root canals), 32 restorations, 50 extractions, and 1 removable partial denture were completed.

\section{Discussion}

It is not surprising that $71 \%$ of the student participants had treated pregnant patients before the OHPDIs, due to the dental clinic being located in a hospital setting that provides $\mathrm{OB}$ care. Future initiatives may consider analyzing students with and without prior experience before an OHPDI event. In addition, intentional efforts were made to increase student exposures and pregnant patients' access to needed dental care as dental training programs serve as entry points to care. ${ }^{3}$ One of the components of this grant initiative, the Double 03T Project: Connecting the OBGYN and Oral Health Care Provider through Team Based Approach Perinatal Care which includes training, teaching, and treating students, $\mathrm{OB}$ residents, and providing patient care, respectively, consisted of providing perinatal oral health care education to pregnant patients in the $\mathrm{OB}$ care wing of the hospital months before the OHPDIs.

Thirty eight percent more (from 63 to $87 \%$ ) of the students reported increased comfort after the OHPDI in treating pregnant patients and more than $75 \%$ reported they are more likely to treat pregnant patients in their practice after graduation. This data suggests that students that receive exposure and clinical experience in treating specific patient groups, such as pregnant patients, become more comfortable in treating pregnant patients and are more likely to treat them in their practice after graduation. The results reflect consistency with the 2005 Dao et al's study that suggests when predoctoral students receive classroom and clinicbased exposure to specific patient groups, they feel more comfortable caring for these patients in their practice. ${ }^{18}$ This is also promising as studies report that clinical practice patterns are sustained postgraduation. ${ }^{19}$

The role of dental and medical education in patient awareness, provider knowledge, and evolution of practice 
cannot be underestimated. ${ }^{20}$ It has been documented that combining didactic and interactive teaching methods is more effective on the learner than either alone, according to a Cochrane review of continuing medical education. ${ }^{21}$ This has also been noted in dental education as well as other areas of health care education. ${ }^{22,23}$ Dental education evidence suggests that student comfort in predoctoral dental programs is a significant predictor of general dentists' stage of readiness to render treatment. ${ }^{20}$ Increasing provider comfort results in 3.4 and 5.8 times more likely to perform these services compared with those contemplating and those unwilling to perform these services, respectively. ${ }^{24}$ These studies were considered in the design of this purposeful hands-on initiative.

The OHPDI is the first documented event to occur in a dental school clinic that provided such an experience. In addition, a substantial number of pregnant women, 34 , were seen for dental treatment in the 2-day event. One previous study reported seeing 55 women, but that was over the course of one academic year. ${ }^{25}$ The 34 women noted in this initiative represents those seen in the 2-day OHPDIs and does not include the additional pregnant women that were seen throughout the academic year.

This study may provide other educational organizations with ideas of how to increase exposure and comfort level of all health care students through interprofessional collaboration to specific patient groups. This study is not without limitations and thus impacts the generalizability of the data presented. Increased comfort level or reported "very comfortable" level of treating pregnant patients in health education settings may not translate to practice behavior of the same reporting students due to other factors that may dictate whether a dentist treats pregnant patients. One factor that may preclude a dentist from treating pregnant patients is the patient's dental insurance coverage. Medicaid beneficiaries have greater challenges in finding dentists that accept their insurance.

Given the sample size, this should be considered as a pilot project. However, the majority of the students reported that because of the OHPDIs they learned how poor oral health may negatively impact pregnancy and birth outcomes and that they are now more likely to treat pregnant patients in their practice.

In conclusion, through interprofessional collaboration, this project positively impacted students and pregnant women. For students, it provided learning, exposure, and experiences in treating pregnant patients resulting in increased knowledge, favorable attitudes, and provider comfort. For pregnant women, it provided opportunities to receive perinatal oral health education and needed dental treatment. Grassroot strategies described could be used for initiatives in other health professions.

\section{Funding}

This was made possible through the generosity of the Detroit Medical Center Foundation, grant \# 2015-0098.

\section{Acknowledgments}

The authors wish to thank Melanie Nevels and Danyelle Vernon, Patient Care Coordinators-University Health
Center, University of Detroit Mercy School of Dentistry, Curles Colbert, DDS, Director of Pre-doctoral Patient Care, University Health Center, University of Detroit Mercy School of Dentistry, Danielle Dunn, Project Coordinator, and Dr. Rose Geist, Associate Professor University of Detroit Mercy School of Dentistry.

The authors also wish to thank Dr. Maria A. Manautou, Lead Dentist Advantage Health Centers-Thea Bowman, who provided feedback on earlier drafts of this manuscript.

\section{References}

1 American College of Obstetricians Gynecologists. American College of Obstetricians Gynecologists Women's Health Care Physicians Committee on Health Care for Unserved Women Committee Opinion No. 569: Oral health care during pregnancy and through the lifespan 2013;122:417-422

2 National Maternal and Child Oral Health Resource Center Georgetown University. Oral health care during pregnancy: A national consensus statement. 2012

3 Lee RS, Milgrom P, Huebner CE, Conrad DA. Dentists' perceptions of barriers to providing dental care to pregnant women. Womens Health Issues 2010;20(05):359-365

4 Mayberry ME, Norrix E, Farrell C. MDA dentists and pregnant patients: a survey of attitudes and practice. J Mich Dent Assoc 2017;99(01):54-62, 83

5 Le M, Riedy C, Weinstein P, Milgrom P. Barriers to utilization of dental services during pregnancy: a qualitative analysis. J Dent Child (Chic) 2009;76(01):46-52

6 Gonik B, Wilson E, Mayberry M, Joarder BY. Pregnant patient knowledge and behavior regarding perinatal oral health. Am J Perinatol 2017;34(07):663-667

7 Giglio JA, Lanni SM, Laskin DM, Giglio NW. Oral health care for the pregnant patient. J Can Dent Assoc 2009;75(01):43-48

8 Iida H. Oral health interventions during pregnancy. Dent Clin North Am 2017;61(03):467-481

9 Curtis M, Silk HJ, Savageau JA. Prenatal oral health education in U.S. dental schools and obstetrics and gynecology residencies. J Dent Educ 2013;77(11):1461-1468

10 Morgan MA, Crall J, Goldenberg RL, Schulkin J. Oral health during pregnancy. J Matern Fetal Neonatal Med 2009;22(09): 733-739

11 Berkowitz RJ. Acquisition and transmission of mutans streptococci. J Calif Dent Assoc 2003;31(02):135-138

12 Boggess KA, Edelstein BL. Oral health in women during preconception and pregnancy: implications for birth outcomes and infant oral health. Matern Child Health J 2006;10(5, Suppl):S169-S174

13 Han YW. Oral health and adverse pregnancy outcomes - what's next? J Dent Res 2011;90(03):289-293

14 Sanz M, Kornman K; working group 3 of the joint EFP/AAP workshop. Periodontitis and adverse pregnancy outcomes: consensus report of the Joint EFP/AAP Workshop on Periodontitis and Systemic Diseases. J Periodontol 2013;84(4, Suppl):S164-S169

15 Xiong X, Buekens P, Goldenberg RL, Offenbacher S, Qian X. Optimal timing of periodontal disease treatment for prevention of adverse pregnancy outcomes: before or during pregnancy? Am J Obstet Gynecol 2011;205(02):111.e1-111.e6

16 Rangel-Rincon LJ, Vivares-Builes AM, Botero JE, AgudeloSuarez A. An umbrella review exploring the effect of periodontal treatment in pregnant women on the frequency of adverse obstetric outcomes. J Evid Base Dent Pract 2018;18 (03):218-239

17 Nascimento MM, Mugayar L, Tomar SL, Garvan CW, Catalanotto FA, Behar-Horenstein LS. The impact of an infant oral health program on dental student's knowledge and attitudes. J Dent Educ 2016;80(11):1328-1336 
18 Dao LP, Zwetchkenbaum S, Inglehart MR. General dentists and special needs patients: does dental education matter? J Dent Educ 2005;69(10):1107-1115

19 Haber J, Hartnett E, Allen K, et al. The impact of oral-systemic health on advancing interprofessional education outcomes. J Dent Educ 2017;81(02):140-148

20 Schroth RJ, Quiñonez RB, Yaffe AB, Bertone MF, Hardwick FK, Harrison RL. What are Canadian dental professional students taught about infant, toddler and prenatal oral health? J Can Dent Assoc 2015;81:f15

21 Forsetlund L, Bjørndal A, Rashidian A, et al. Continuing education meetings and workshops: effects on professional practice and health care outcomes. Cochrane Database Syst Rev 2009;15(02):CD003030
22 Fein JE, Quinonez RB, Phillips C. Introducing infant oral health into dental curricula: a clinical intervention. J Dent Educ 2009;73(10): 1171-1177

23 Morgan PJ, Cleave-Hogg D. Comparison between medical students' experience, confidence and competence. Med Educ 2002; 36(06):534-539

24 McFarland T, Quinonez R, Phillips C, Lee J, Chung Y. Provision of preventive oral health services to infants and toddlers: North Carolina general dentists' readiness. J Oral Health Comm Dent 2013;7:140-147

25 Jackson JT, Quinonez RB, Kerns AK, et al. Implementing a prenatal oral health program through interprofessional collaboration. J Dent Educ 2015;79(03):241-248 